\title{
Mucosal Malignant Melanoma of Nasal Bauity
}

Girija Chate, James Thomas, Priya Shah, SC Deogaonkar, Paresh Chavan

From the Department of ENT and HNS, Padmashree Dr. D. Y. Patil Medical

College, Pimpri, Pune 411018 , India.

\begin{abstract}
:
Mucosal malignant melanoma of the nasal cavity and paranasal sinuses is a rare entity. It is an unusual cause of nasal obstruction and epistaxis seen especially in elderly. This tends to be an aggressive tumor with poor prognosis because of distant metastasis and loco-regional recurrence. Surgical excision is the preferred treatment modality with adjuvant radiotherapy for selected cases with large bulky primary disease or regional lymph node metastasis. Immunotherapy is showing some promise and chemotherapy at present has a role only in palliation. Here we present a case of mucosal malignant melanoma in a 62 years old female that was treated by wide local excision after ruling out distant and local metastasis.
\end{abstract}

Key words: Melanoma, Nasal cavity, Epistaxis, Nasal Obstruction, Nose Neoplasms.

\section{Introduction}

Incidence of malignant melanoma as a whole is $2: 100,000$. Out of these, mucosal malignant melanomas of head and neck account for less than 1 $\%$ of these cases [1]. Mucosal malignant melanoma of the nose and paranasal sinuses is extremely rare. Prognosis is generally poor and unpredictable. Early diagnosis confirmed by immunohistochemistry [2] and radical surgical management appears to offer the best hope for curing the disease.

Malignant melanomas may be misdiagnosed as a polyp or an inverted papilloma, especially when amelanotic. Histopathology report with immunohistochemistry gives accurate diagnosis. We report clinical and histopathological features of a case of mucosal malignant melanoma of nasal cavity in a 62-year-old female patient.

\section{Case Report}

A 62-year-old female patient came to the outpatient department of otolaryngology with swelling in the left nasal cavity causing obstruction to breathing and gradually increasing in size over one year. There were occasional episodes of epistaxis, few drops at a time. On clinical examination, the swelling was reddish pink with brownish discoloration at places, soft in consistency with irregular surface filling up the whole of left nasal cavity. On anterior rhinoscopy, turbinates could not be visualized. The mass was bleeding on manipulation. There was no pigmented lesion elsewhere. Clinically, there was no

\section{Corresponding Author: Dr. Girija Ghate}

Email: girija.ghate@gmail.com

Received: July 4, 2013 | Accepted: July 31, 2013 | Published Online: August 30, 2013

This is an Open Access article distributed under the terms of the Creative Commons Attribution License (creativecommons.org/licenses/by/3.0)

Conflict of interest: None declared | Source of funding: Nil | DOl: http://dx.doi.org/10.17659/01.2013.0069 
regional lymphadenopathy which was confirmed by ultrasound neck.

CT scan of the paranasal sinuses showed a soft tissue mass occupying whole of the left nasal cavity. There was no bony erosion or paranasal sinus involvement. Septum was deviated to right side [Fig.1]. A biopsy of the lesion was done with a clinical suspicion of inverted papilloma/carcinoma.

With a left lateral rhinotomy incision, the mass was exposed. It was very soft and friable and showed attachment to mucosa of superior part of the septum and at one place on the floor. The turbinates appeared to be normal. Wide local excision of the mass with cauterization of the base was done. The specimen was sent for histopathology. Postoperative period was uneventful. The wound healed well and suture removal was done on seventh day. After receiving histopathology of malignant melanoma, she was subjected to ultrasound abdomen to rule out distant metastasis in the liver.

On histopathology, the tumor tissue showed different growth patterns. Tumor cells were oval to spindle shaped growing in solid fascicles, whorls, ribbons and reticulated pattern. There was mucosal infiltration seen. Occasional cells showed brownish pigment most probably melanin.

Immunohistochemistry revealed tumor cells to be S-100 and HMB 45 positive and negative for EMA, CEA, MHW-CK and SMA. This gave the definitive diagnosis of Mucosal Malignant Melanoma.

\section{Discussion}

Malignant melanomas are neural crest-derived neoplasms originating derived from malignant transformation of melanocytes in the basal layer of mucosa. First case of sinonasal malignant melanoma was reported by Lucke in 1869 and has been reported in about $2-8 \%$ of sinonasal tumors.

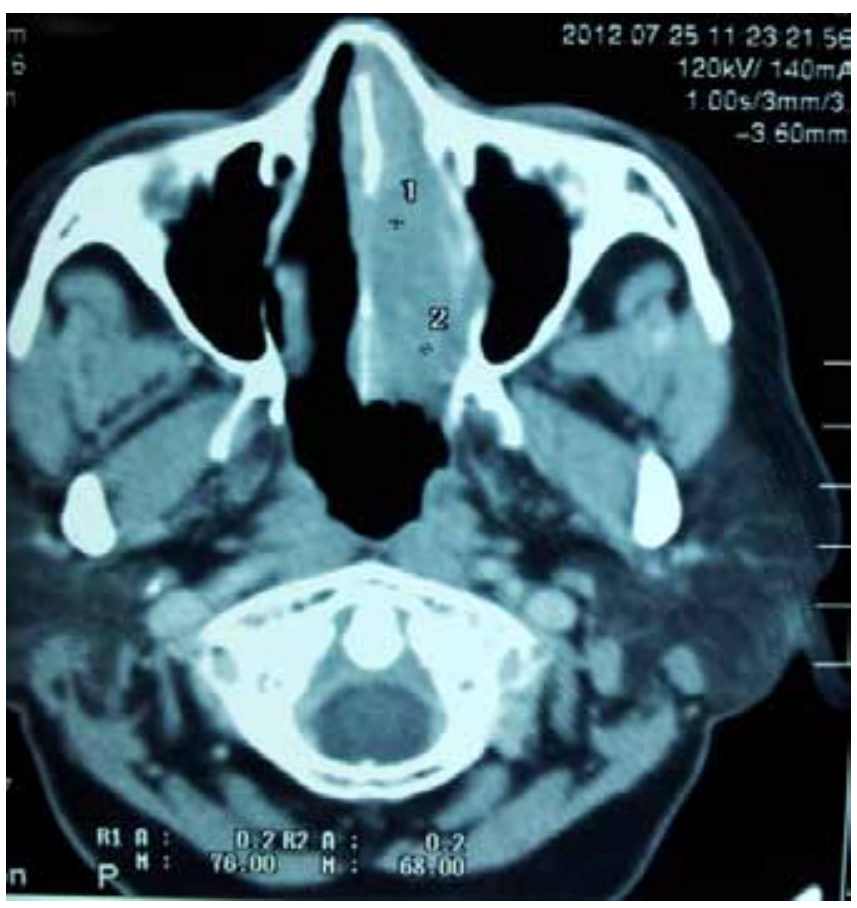

Fig.1: CT scan of the paranasal sinuses showing a soft tissue mass occupying whole of the left nasal cavity. There is no bony erosion or paranasal sinus involvement.

Of the head and neck malignant melanomas, over $90 \%$ are cutaneous, $5 \%$ ocular, $2.2 \%$ peripheral unknown primaries and only $1.3 \%$ are mucosal [1]. Mucosal malignant melanoma (MMM) of the upper aerodigestive tract (UADT) represents $0.5-3 \%$ of malignant melanomas of all sites. UADT MMMs are more common in men than in women [2]. Mucosal melanomas are far more aggressive than cutaneous ones and have a much poorer outcome.

In the nasal cavity, the most frequent site of occurrence is the nasal septum (anterior portion) and the lateral nasal wall. Clinically most patients have symptoms of nasal obstruction or epistaxis or both. On examination, nasal melanomas tend to be large, bulky, friable mass which bleed with manipulation. Clinical appearance of the tumors may be indistinguishable from benign polyposis, especially in amelanotictumors. The nasal melanomas project 
into the involved cavity and may have a somewhat polypoidal configuration. The consistency is firm, friable or gelatinous. Incidence of regional lymph node metastasis on presentation is about 5 to $15 \%$ with submandibular nodes being most commonly affected. About $10 \%$ of the patients may have distant metastasis on presentation [3].

Early diagnosis requires high index of suspicion. Definitive diagnosis is confirmed only by immunohistochemistry of the surgical specimen with tumor being S-100 and HMB 45 positive [2]. Because of its rarity, there can be some differences in opinions regarding preferred modality of treatment. Surgery in the form of wide local excision remains the choice of treatment today. Different surgical procedures like lateral rhinotomy, maxillectomy, total rhinectomy or craniofacial resection are done depending on the extent of the disease.

Historically, mucosal malignant melanoma was characterized as a radioresistant disease. But some studies have shown that post operative radiotherapy may give better local control. It should be used as adjuvant therapy in cases with regional metastasis or large bulky primary disease [4]. Radiotherapy alone was reported to give absolute local control in $61 \%$ cases by Gillian et al. [5].

Mucosal malignant melanoma is relatively chemoresistant tumor. Palliative chemotherapy is used in advanced disease with dacarbazine being the chemotherapeutic agent. Immunotherapy has shown a great promise in the treatment of this disease in recent studies. OK 432, Interleukin 2, lymphokine activated killer cells, BCG (Bacilli Calmette Guerin) vaccines have shown partial success but further trials in this regards are needed $[6,7]$.

MMM tends to aggressive tumor and overall prognosis and survival rate ranges between 10 to $40 \%$ with mean survival being $21-24$ months [8].
Poor prognostic factors include local and distant metastasis, local recurrence, vascular invasion and a second primary [9]. Single most powerful predictor is absence of regional lymph nodes. Other contributing factors for poor prognosis are delay in detection and inaccurate histological diagnosis because of its rarity and low index of suspicion [10].

Our patient came with history of nasal obstruction and epistaxis. A nasal mass was seen protruding from the left nasal cavity. After appropriate investigations, she was subjected to surgery and the histopathology report with immunohistochemistry gave the diagnosis of malignant melanoma. Surgery being the first treatment option, this patient was subjected to surgery. She is under regular follow ups considering the high rate of recurrence.

\section{References}

1. Jones Andrew S. Mucosal malignant melanoma. In: Scott Brown's Otorhinolaryngology, Head and Neck Surgery. 7th ed. Vol.2Hodder Arnold: UK; 2008: 2406-2416.

2. Bothale KA, Maimoon SA, Patrikar AD, Mahore SD. Mucosal malignant melanoma of the nasal cavity. Indian J Cancer. 2009;46:67-70.

3. Dwivedi R, Dwivedi R, Kazi R, Kumar S, Agarwal SP. Mucosal melanoma of nasal cavity and paranasal sinus. J Can Res Ther. 2008;4:200202.

4. Harwood AR, Cummings BJ. Radiotherapy for mucosal melanomas. Int J Radiat Oncol Biol Phys. 1982;8:1121-1126.

5. Gilligan D, Slevin NJ. Radical radiotherapy for 28 cases of mucosal melanoma in nasal cavity and sinuses. Br J Radiol. 1991;64:1 147-1 150.

6. Mundra RK, Sikdar A. Endoscopic removal of malignant melanoma of nasal cavity. Ind J Otolaryngol Head Neck. 2005;57:341-343.

7. Sober AJ, Koh HK, Wittenberg, Washington $C \vee$ Jr. Melanoma and other skin cancer. In: Braunwald E, Hauser SL, editors. Harrison's 
Principles of Internal Medicine. 15th ed. Vol. 1: USA:Mcgraw Hill 2001: pp.554-559.

8. Conley JJ. Melanomas of the mucous membrane of the head and neck. Laryngoscope 1989; 1 2:1248-1254.

9. Prashant D, Kujur P, Sharma N, Prashant V. Primary Oral Malignant Melanoma. Journal of
Case Reports. 2013;3:272-276.

10. Errarhay S, Mamouni N, Mahmoud S, El fatemiH, Saadi H, MesbahiO et al. Primary Malignant Melanoma of the Female Genital Tract: Unusual Localization. Journal of Case Reports. 2013;3:169-175. 